\title{
Evaluation of IMproving Palliative Care Education and Training Using Simulation in Dementia (IMPETUS-D) a Staff Simulation Training Intervention to Improve Palliative Care of People with Advanced Dementia Living in Nursing Homes: A Cluster Randomised Controlled Trial
}

Joanne Tropea ( $\sim$ Joanne.Tropea@mh.org.au )

University of Melbourne

Debra Nestel

Monash University

Christina E Johnson

Monash University

Barbara J Hayes

Northern Health

Anastasia F Hutchinson

Deakin University

Caroline A Brand

University of Melbourne

Brian H Le

Royal Melbourne Hospital

Irene Blackberry

La Trobe University

Gideon A Caplan

Prince of Wales Hospital

Ross Bicknell

Royal Melbourne Hospital

Graham Hepworth

University of Melbourne

Wen K Lim

Royal Melbourne Hospital 
Keywords: End-of-life care, Palliative care, Dementia, Staff training, Nursing homes

Posted Date: November 12th, 2021

DOI: https://doi.org/10.21203/rs.3.rs-1001512/v1

License: (c) (1) This work is licensed under a Creative Commons Attribution 4.0 International License. Read Full License 


\section{Abstract}

\section{Background}

Access to palliative and end-of-life care for people with dementia living in nursing homes is suboptimal. Nursing home staff require training in palliative care for dementia to equip them with knowledge and skills to deliver high quality care.

Objective

The primary aim was to evaluate the effectiveness of a simulation training intervention (IMPETUS-D) aimed at nursing home staff on reducing unplanned transfers to hospital and/or deaths in hospital among residents living with dementia.

Design

Cluster randomised controlled trial of nursing homes with process evaluation conducted alongside.

Subjects \& setting

1304 people with dementia living in 24 nursing homes (12 intervention/12 control) in three Australian cities, their families and direct care staff.

Methods

Randomisation was conducted at the level of the nursing home (cluster). The allocation sequence was generated by an independent statistician using a computer-generated allocation sequence.

Staff from intervention nursing homes had access to the IMPETUS-D training intervention, and staff from control nursing homes had access to usual training opportunities. The predicted primary outcome measure was a $20 \%$ reduction in the proportion of people with dementia who had an unplanned transfer to hospital and/or death in hospital at 6-months follow-up in the intervention nursing homes compared to the control nursing homes.

Results

At 6-months follow-up, 128 (21.1\%) people with dementia from the intervention group had an unplanned transfer or death in hospital compared to $132(19.0 \%)$ residents from the control group; odds ratio 1.14 $(95 \% \mathrm{Cl}, 0.82-1.59)$. There were suboptimal levels of staff participation in the training intervention and several barriers to participation identified.

\section{Conclusion}

This study of a palliative dementia care staff training intervention found no difference in the proportion of residents with dementia who had an unplanned hospital transfer. Implementation of the intervention 
was challenging and likely did not achieve adequate staff coverage to improve staff practice or resident outcomes.

Trial registration:

Australian New Zealand Clinical Trials Registry (ANZCTR): ACTRN12618002012257. Registered $14 / 12 / 2018$.

\section{Background}

For many people with advanced dementia, the last months or years of life are spent in nursing homes [1]. However, there are reports of suboptimal palliative and end-of-life dementia care, including unnecessary transfers to hospital, burdensome interventions, and poor pain management [2-4]. It has been identified that a major contributory factor to this includes low levels of staff education and skills in provision of palliative and end-of-life care $[5,6]$.

Training staff about palliative care in dementia is a recognised strategy to improve the quality of care provided $[6,7]$. Simulation is an immersive training technique that attempts to evoke real-life scenarios to promote deeper learning and better transfer of skills to work. There is a growing evidence base to support the benefits of simulation in healthcare education, but very few studies set in nursing homes and no controlled trials on dementia-specific palliative care [8]. Among the few studies that have assessed palliative dementia care training in nursing homes, two used simulation techniques but were single-site, non- randomised studies and of low methodological quality [9-13].

This study evaluated a training program IMproving Palliative care Education and Training Using Simulation in Dementia (IMPETUS-D) for nurses and personal care workers (PCWs), aimed at improving the quality of palliative care provision and outcomes for people with dementia living in nursing homes. The study used a cluster randomised controlled trial (RCT) design in parallel with a process evaluation. The primary aim was to evaluate the effectiveness of IMPETUS-D training on reducing unplanned transfers to hospital and/or deaths in hospital among residents living with dementia. The study tested the hypothesis that nursing homes that participated in the IMPETUS-D training intervention would reduce the rate of unplanned transfers to hospital and or deaths in hospital among residents with dementia by $20 \%$ compared to the control nursing homes.

\section{Methods}

The trial was registered (ANZCTR: ACTRN12618002012257) and conducted according to a pre-defined published protocol to minimise potential biases, and subsequent deviations are reported [14]. Research methods and reporting were in accordance with the CONSORT 2010 statement: extension to cluster randomised trials [15]. Ethics approval was received by the Melbourne Health Human Research and Ethics Committee (HREC/17/MH/336). 


\section{Trial design}

A multi-centre, cluster RCT design with an associated process evaluation was undertaken in Australia. Study sites were nursing homes (the clusters), that met the inclusion criteria of having at least 20 people with dementia permanently living in the care home and requiring high level care. A single private aged care provider managed all facilities. The study ran from December 2018 to October 2020. All nursing homes entered the study in December 2018 and baseline data collection commenced, staff from all the intervention nursing homes received access to the training intervention in early April 2019, the follow-up period commenced 1 October 2019 to 30 September 2020.

\section{Participants and recruitment}

The study involved 24 private nursing homes in Sydney, Melbourne and Adelaide, Australia. To be eligible for inclusion in the study, nursing homes had to have a minimum of 20 people with dementia living permanently in the care home and requiring high level care. All permanent care home residents with a recorded diagnosis of dementia were included.

People with dementia were defined as having a diagnosis of dementia if this was documented in residents' nursing home files by a medical practitioner or nurse. Nurses and PCWs who worked at the nursing homes were recruited to participate, with additional promotion and information about the study provided face-to-face at staff meetings, and via flyers in staff rooms. Plain language statements were provided in hard copy and electronically to all potential staff participants, and consent was obtained from staff who participated in surveys and interviews. Participation in research activities and the training was voluntary.

Bereaved family members of residents with dementia who died during the follow-up period were invited to participate in a survey. The invitation was posted 6-8 weeks after the death of a resident. If no response was received, one follow-up phone call was made to check the survey had been received, to further explain the project and remind families to participate if they wished.

\section{Intervention}

The IMPETUS-D training program (intervention) was developed for the study by experts in simulation and healthcare worker education. The program comprised 11 modules covering key aspects of best practice palliative and end-of-life care for people with dementia living in nursing homes (Box 1). Modules include case studies with short video scenarios filmed in authentic care home settings. These videos were used as the basis for questions to stimulate learning and practicing 'what to say' in different situations, for example, when family are worried about a resident not eating towards the end of life. The length of modules ranged from 15 to 45 minutes. 
Box 1 - Modules and topics covered

- Module 1 describes the natural progression of dementia and how to recognise when deterioration is due to dementia versus delirium.

- Module 2 focuses on caring for a person with advanced dementia when they are approaching the end of their life.

- Module 3 discusses the reasons why people with advanced dementia are typically best cared for in the care home and describes the challenges people with advanced dementia face in the busy hospital environment.

- Module 4 introduces Goals of Care (GOC) plans, and highlights that finding out what's important to the person is vital to providing best care.

- Module 5 describes what is needed for high quality and best practice GOC plan.

- Module 6 discusses pain and the challenges in managing pain in people with advanced dementia.

- Module 7 focuses on recognising the dying phase, including reduced eating and drinking, and helping families understand that reduced eating and drinking at the end of life is normal.

- Module 8 discusses focusing on what is most important to the person when they are dying and ways to keep a person as comfortable as possible. It also describes terminal restlessness a common end of life symptom, and how care staff can recognise and manage it.

- Module 9 focuses on changes in breathing at end of life, what to expect as death approaches and ways to support the family. It also discusses the concerns care staff may have as a resident approaches death and includes reflection of learners' real experiences.

- Module 10 raises the importance of communicating openly, honestly and frequently with residents and their family members.

- Module 11 shifts the focus of caring from residents to care providers. It encourages carers to consider their own feelings about death and dying and how they manage these feelings when a resident is at the end of their life and then dies.

The training could be accessed online via desktop computer, laptop, tablet, or smartphone. PCWs were expected to complete five core modules - Modules 1-3 and 10, and one symptom management module (Modules 6-9). The remaining modules were recommended. Nurses were expected to complete the two goals of care (GOC) modules and the communication module, and the remaining modules were recommended. As an incentive staff were paid for their time if they participated in the training, and other strategies were used to promote staff participation (Box 2).

\section{Control}

Staff from nursing homes in the control group did not receive the IMPETUS-D training but had access to usual training opportunities. 
Box 2 - Strategies used to encourage staff recruitment and participation in IMPETUS-D training

- Project nurse consultants employed by the provider were recruited in each city to assist with implementation and data collection activities

- General managers, nurses and PCWs were sent emails by the research team and the provider executive to inform them about the project, and flyers were put up on staff noticeboards at each care home

- Staff were paid for their time if they participated in the training (face-to-face sessions or online)

- The research team held face-to-face sessions at each care home

- Project nurse consultants and / or research team visited nursing homes to promote the training and spoke with staff in-person individually, in small groups, or at staff meetings

- Other incentive: the nursing home with the highest proportion of staff to participate in the training won a food hamper

\section{Outcomes}

Outcome measures pertain to the cluster or nursing home level. The primary outcome measure was the proportion of residents with dementia who had an unplanned hospital transfer and/or death in hospital during the 6-months follow up. Secondary outcomes measures were: hospital transfers or deaths in hospital over 12-months among residents with dementia, uptake of GOC plans over 6 and 12-months for residents with dementia, change in staff knowledge and attitudes about palliative care for people with dementia at 6 months follow up, and bereaved family satisfaction with care.

\section{Randomisation and blinding}

Randomisation was conducted at the level of the nursing home (cluster). The allocation sequence was generated by an independent statistician using a computer-generated allocation sequence. To ensure equal numbers of nursing homes per study arm, the randomisation used blocks of 4 , stratified by city and facility size (small or large, based on the total number of residents). To preserve blinding, the randomisation list was stored and maintained in a secure location by the statistician who was independent from trial recruitment or training.

\section{Data collection}

Details of the data collection for outcome measures can be found in the protocol paper [14]. A dedicated study database was developed using the Research Electronic Data Capture (REDCap) tools hosted at the University of Melbourne [16]. Baseline resident demographic and clinical information, and follow-up resident-level outcomes (hospital transfers and deaths) were collected by project nurse consultants (not blinded to allocation) from the residents' care home records or extracted from the provider's database.

At baseline and 6-months follow-up, care home staff were invited to complete a survey which included demographic information and the Questionnaire on Palliative Care for Advanced Dementia (qPAD) [17]. The qPAD is a 2-part instrument consisting of a 23-item knowledge test and 12-item attitude scale. It was used to measure the secondary outcomes of staff knowledge and attitudes towards providing palliative 
dementia care. A total qPAD score (range 12-83) was derived by adding knowledge test (range 0-23) and attitude scale (range 12-60) scores; higher scores indicating better knowledge and more positive attitudes.

During the follow-up period, bereaved families' perception of care was assessed using the Satisfaction With Care at the End-of-Life Dementia (SWC-EOLD) scale [18]. The SWC-EOLD assessed satisfaction with care during the last 90 days of life, and consisted of 10 items, each measured on a 4-point Likert scale defined as 1 =strongly disagree, to $4=$ strongly agree. The total SWC-EOLD score was calculated by adding all 10 items (range 10-40); higher scores indicated greater satisfaction.

\section{Sample size and power calculations}

An a-priori sample size calculation was undertaken as per protocol [14]. The power analyses were based on the following assumptions: proportion of composite events (hospital transfer and/or death in hospital) of 0.65 and 0.85 over 6 months of follow-up in the intervention and control groups respectively, two-sided significance level of 0.05 (alpha). We estimated that 12 clusters (nursing homes) in each study arm, with 20 - 25 residents with dementia in each cluster, was needed with an Intra-Cluster Correlation (ICC) of 0.05 . With these assumptions, the minimum power to observe a difference of proportion by 0.20 between the intervention and control group is $94 \%$.

\section{Statistical analysis}

Descriptive statistics were used to summarise baseline care home and resident-level characteristics. Analyses of primary and secondary outcomes were on an intention to treat (ITT) basis, with the fundamental principles of analysing data from cluster RCTs followed. To compare the proportion of composite events for the primary outcome, logistic regression models were used with standard errors weighted by the cluster effects, clusters being the nursing homes. Estimated odds ratio (OR) and associated $95 \%$ confidence interval are presented, along with the $p$ value for testing the hypothesis that the OR is 1. Total scores from the $\mathrm{qPAD}$ and SWC-EOLD were considered as a continuous variable. Mean scores were compared between the groups using mixed models which adjust for cluster effect. Statistical analyses were conducted in Stata MP version 15 [19]. All statistical tests were two-sided and the significance level was set at 0.05 .

\section{Process evaluation}

Process evaluation was used to assess the implementation of the training intervention, to explore barriers and facilitators that influence implementation of the intervention, as well as the intervention mechanisms and outcomes. Measures included staff participation and module completion (reach and dose), participants' experience, and barriers to participation.

The a-priori aim was for $80 \%$ of staff to complete the core modules for their discipline. This was amended during the training period to $50 \%$ of nurses in response to feedback that staff had other mandated training occurring at the same time. Data on the number of staff who participated in the 
training, the number of modules, and the number of core modules commenced and completed was collected from the learning management system (LMS) and from attendance records at each face-to-face training session.

Data on participants' experience and satisfaction with the intervention was collected from feedback items at the end of each module, and at 6-month follow-up staff interviews and follow-up staff surveys. Data from the research team notes and log of activities, informal feedback from staff, follow-up staff surveys, and staff interviews was used to identify factors that may have influenced reach, dose, participant satisfaction and barriers to participation.

Qualitative data from the surveys and interviews were imported from Microsoft Excel and Microsoft Word files into NVivo 12 for coding [20]. Data was analysed by the research team and key themes identified based on anticipated and emergent themes [21]. An iterative process was used, with coding trees and summarised data presented to members of the IMPETUS-D Project Working and Advisory Group for review and input.

\section{Results}

Of the 44 nursing homes, 27 were eligible for inclusion. To obtain balance across locations and size, 24 nursing homes were randomised into the intervention or control group (Figure 1). All 24 agreed to participate in the study, and all remained involved in the study throughout the project timeline.

\section{Residents with dementia}

At baseline, among all residents living in the 24 nursing homes 1304 (59.8\%) had a diagnosis of dementia recorded; 608 in the intervention nursing homes and 696 in the control nursing homes. Demographic and clinical data of residents with dementia were similar for those living in the intervention and control homes, except more residents from the intervention group had an advance care directive (ACD) in place (Table 1).

\section{Table 1: Baseline clinical and demographic characteristics of residents with dementia}




\begin{tabular}{|c|c|c|c|}
\hline Characteristic & $\begin{array}{l}\text { Intervention nursing } \\
\text { homes }(n=608)\end{array}$ & $\begin{array}{l}\text { Control nursing homes } \\
(n=696)\end{array}$ & $\begin{array}{l}\text { Total } \\
(\mathrm{N}=1,304)\end{array}$ \\
\hline Age in years, median (IQR) & $86.4(81.0-91.5)$ & $87.3(80.9-91.5)$ & $\begin{array}{l}86.9(80.9- \\
91.5)\end{array}$ \\
\hline Female, n (\%) & $409(67.3)$ & $463(66.5)$ & $872(66.9)$ \\
\hline $\begin{array}{l}\text { Years living in care home, } \\
\text { median (IQR) }\end{array}$ & $2.2(1.0-4.6)$ & $2.3(0.9-4.2)$ & $\begin{array}{l}2.3(0.9- \\
4.4)\end{array}$ \\
\hline \multicolumn{4}{|l|}{ ACFI scores - high, n (\%) } \\
\hline Activities of Daily Living & $342(56.3)$ & $425(61.1)$ & $767(58.8)$ \\
\hline Behaviour & $468(77.0)$ & $556(79.9)$ & $1024(78.5)$ \\
\hline Complex health care needs & $416(68.4)$ & $502(72.1)$ & $918(70.4)$ \\
\hline ACD in place, $n(\%)$ & $198(32.6)$ & $190(27.3)$ & $388(29.8)$ \\
\hline \multicolumn{4}{|l|}{ Type of dementia } \\
\hline Alzheimer's disease & $284(46.4)$ & $346(49.7)$ & $628(48.2)$ \\
\hline Vascular dementia & $67(11.0)$ & $87(12.5)$ & $154(11.8)$ \\
\hline Mixed dementia & $56(9.2)$ & $53(7.6)$ & $109(8.4)$ \\
\hline Lewy body dementia & $14(2.3)$ & $12(1.7)$ & $26(2.0)$ \\
\hline Fronto-temporal dementia & $11(1.8)$ & $10(1.4)$ & $21(1.6)$ \\
\hline Other & $20(3.3)$ & $30(4.3)$ & $50(3.8)$ \\
\hline Not specified & $158(26.0)$ & $158(22.7)$ & $316(24.2)$ \\
\hline \multicolumn{4}{|l|}{ Comorbidities } \\
\hline CHF & $68(11.2)$ & $92(13.2)$ & $160(12.3)$ \\
\hline Chronic lung disease & $76(12.5)$ & $92(13.2)$ & $168(12.9)$ \\
\hline Chronic kidney failure & $51(8.4)$ & $69(9.9)$ & $120(9.2)$ \\
\hline Chronic liver disease & $6(1.0)$ & $4(0.6)$ & $10(0.8)$ \\
\hline Stroke & $102(16.8)$ & $122(17.5)$ & $224(17.2)$ \\
\hline Cancer & $109(17.9)$ & $115(16.5)$ & $224(17.2)$ \\
\hline
\end{tabular}

Abbreviations: ACD advance care directive; ACFI Aged care funding instrument; CHF congestive heart failure; IQR inter quartile range

Unplanned hospital transfers and deaths in hospital are shown in Table 2. During 6-months follow-up, a total of $260(19.9 \%)$ residents with dementia from all care homes had at least one unplanned hospital 
transfer; $128(21.1 \%)$ in the intervention and $132(19.0 \%)$ in the control group. Analysis of the primary outcome (proportion of hospital transfers or deaths in hospital among residents with dementia over 6 months) yielded an odds ratio of $1.14(95 \% \mathrm{Cl}, 0.82-1.59), \mathrm{p}=0.44$. Overall, 154 residents died during 6months, $75(12.3 \%)$ from the intervention and 79 (11.4\%) from the control group.

During 12-months follow-up, 407 (31.2\%) residents with dementia had at least one unplanned transfer to hospital, 201 (33.1\%) from the intervention and 206 (29.6\%) from the control group (odds ratio 1.17; 95\% $\mathrm{Cl}, 0.84-1.63)$; and 310 (23.8\%) residents died, 154 (25.3\%) from the intervention and 156 (22.4\%) from the control group.

\section{Table 2: Number and proportion of residents with dementia with an unplanned hospital transfer and death in hospital at 6- and 12-months follow-up ( $N=1304)$}

\begin{tabular}{|llll|}
\hline Outcomes, $\mathrm{n}(\%)$ & $\begin{array}{l}\text { Intervention } \\
(\mathrm{n}=608)\end{array}$ & $\begin{array}{l}\text { Control } \\
(\mathrm{n}=696)\end{array}$ & Odds ratio $(95 \% \mathrm{Cl}), \mathrm{p}$ value \\
\hline Hospital transfers, 6 months & $128(21.1)$ & $132(19.0)$ & $1.14(0.82-1.59), \mathrm{p}=0.44$ \\
\hline Hospital transfers, 12 months & $201(33.1)$ & $206(29.6)$ & $1.17(0.84-1.63), \mathrm{p}=0.34$ \\
\hline Deaths in hospital, 6 months & $14(18.7)$ & $14(17.7)$ & $1.07(0.39-2.91), \mathrm{p}=0.90$ \\
\hline Deaths in hospital, 12 months & $22(3.6)$ & $28(4.0)$ & $0.90(0.44-1.83), \mathrm{p}=0.76$ \\
\hline
\end{tabular}

Two of the core nursing modules focused on GOC discussions and documenting GOC medical treatment plans [22]. A new GOC form was introduced in the module and all senior nurses and general managers were sent a copy of the form. However, there was no uptake of the form which relied on a doctor for completion. This was despite general practitioners being sent information about the forms.

\section{Staff knowledge and attitudes}

A total of 330 staff completed the follow-up qPAD, 122 from the intervention and 208 from the control nursing homes. Staff from both groups were similar in terms of sex, age, position, years' of aged care experience, hours worked per week, and highest level of education. (Appendix 1). Among them, 218 staff completed both baseline and follow-up qPAD, 96 from the intervention and 122 from control nursing homes.

There was no significant between-group difference in total qPAD scores; intervention group mean score $=60.1$ (95\% Cl 57.4-62.8) compared to the control group mean score=59.5 (95\% Cl 57.2-61.8), $p=0.77$. Most of the variability was contributed by individuals (93\%) not nursing homes (7\%) indicating clustering did not have a big effect on scores. Similarly, when baseline scores were accounted for, there was no significant between-group difference in total qPAD scores; adjusted intervention group mean score $=61.1$ and control group mean score $=60.0(p=0.53)$. 


\section{Bereaved carer survey}

A total of 72 of 258 bereaved carer surveys were received over 12-months. At the request of the provider, surveys were not sent if nursing homes had experienced COVID outbreaks or if coroner's investigation was underway. There were no significant between group differences in mean SWC-EOLD scores, with mean scores of 26.6 in the intervention and 27.0 in the control group, $p=0.80$. Most of the variability was contributed by individuals (87\%) not nursing homes $(13 \%)$ demonstrating that clustering did not have had a big effect on SWC-EOLD score.

\section{Process evaluation findings}

Due to low uptake of the online modules and feedback from staff, the training period was extended from 2 months as originally planned to 6 months, and face-to-face training sessions were held by members of the research team and the project consultant nurses at each of the intervention sites to boost training participation.

Reach and dose: During the 6-month training period 42 (4.3\%) staff completed their core modules, 27 (3.7\%) PCWs and 15 (6.0\%) nurses. None of the nursing homes achieved the aim of $50 \%$ of nurses completing core modules. The three nursing homes with the highest proportion of nurses completing core modules were $24 \%$ (care home 9), 21\% (care home 4) and 15\% (care home 3 ) (Figure 2).

Overall, 251 (26\%) staff completed at least one module during the training period, 79 nurses and 172 PCWs. 160 staff attended the face-to-face sessions, and 102 participated in the training online, with some staff completing modules both online and at face-to-face sessions. The proportion of staff from each care home who participated in the training ranged from $15 \%$ to $53 \%$.

Staff satisfaction with the training: Among the 103 follow-up survey respondents who had done the training, 61 (59\%) agreed that completing the modules increased their confidence in looking after residents with dementia, $12(12 \%)$ did not agree or were unsure and $30(29 \%)$ did not respond. The reasons staff gave for not participating in the training included: $25 \%$ were unable to attend the session, $17 \%$ were not aware of the training, $16 \%$ had technical issues accessing the training online, and $15 \%$ did not have time.

Barriers to participation: The Consolidated Framework for Implementation Research (CFIR) was used to design the process evaluation and analyse the findings from the qualitative interview and survey data [23]. One of the main barriers to staff participation was competing priorities that occurred at the same time as the IMPETUS-D program was implemented, specifically the introduction of the National Aged Care Standards and the Royal Commission into Aged Care Quality and Safety. Staff were expected to attend training in preparation for the new standards, and many had additional administrative work in relation to the new standards and the Royal Commission, and these activities took time and the focus away from IMPETUS-D. These barriers relate to the CFIR constructs of relative priority (standards took priority over IMPETUS-D training) and available resources (staff not having adequate or dedicated time 
and resources to do the training). In addition, there were organisational and nursing home level barriers, that affected implementation including variable leadership engagement, intra-organisational communication, turnover of care home management and senior nurse roles at $50 \%$ of intervention homes (stability of the team), and time constraints and disruption to schedule. Some characteristics of the training intervention itself were also reported as potential barriers to implementation including the online and individual learning approach, the use of a separate LMS rather than the organisation's LMS that staff were familiar with, and that the training was not mandatory.

\section{Discussion}

The IMPETUS-D simulation-based staff training program on quality palliative and end-of-life care in dementia had no effect on hospital transfers and deaths in hospital of nursing home residents with dementia, staff knowledge and attitudes, or bereaved family satisfaction with care. Implementation of the intervention was suboptimal with only $26 \%$ of staff participating in the program.

\section{Comparison with other studies}

Our systematic review of five controlled studies found there is a lack of evidence to support simulation training in non-cancer palliative care for healthcare workers [8]. Improvements in staff communication skills, knowledge acquisition and attitudes towards death and comfort were reported in three studies, but these studies had several methodological limitations. Further, one good quality RCT was unable to show any change in clinician practice or patient outcomes. These findings highlighted the need for more rigorous research to evaluate the effect of simulation training in this clinical field, in particular the impact on practice change and patient outcomes.

Two recent cluster RCTs have evaluated palliative care training programs in nursing homes [24, 25]. However, these studies did not focus on dementia-specific palliative care. The European multi-country PACE study assessed an intervention that used a train-the-trainer approach to integrate non-specialist palliative care in 37 nursing homes compared to 36 controls [25]. They were unable to show improvements in residents' comfort at end-of-life, and despite improvements in intervention staff knowledge compared to the controls, the difference was not clinically relevant. The process evaluation found high variability in the implementation of the intervention, and reported the main reasons related to the program itself and its delivery, the PACE trainer, and organisational issues [26].

The study by Lamppu et al. (2021) assessed the effectiveness of staff training in palliative and end-oflife care in 20 Helsinki nursing homes [24]. The intervention training was based on a palliative care training-needs survey and this likely contributed to the high participation rates (74\%). However, they were unable to show any between-group differences in residents' health-related quality-of-life, hospital admissions or emergency presentations. They attributed the null findings to potential poor selection of quality-of-life instrument, lower than expected deaths, and different learning needs of professions; and concluded that training interventions alone might be insufficient to produce improvements in practice or outcomes. 
Both these studies were unable to show the training led to improvements in resident outcomes. Further studies may have to incorporate interventions beyond education such as use of the protocols to improve palliative care outcomes.

\section{Strengths and limitations}

Strengths of the IMPETUS-D study include its large sample size. multi-site design and use of an innovative simulation-enhanced program about the specific palliative care needs of people with dementia. All 24 nursing homes recruited to the study, participated in the research and all intervention nursing homes participated in the training. However, there were several study limitations.

The degree of staff participation was lower than expected and a key limitation to this study.

One of the biggest issues was the timing of the implementation of the intervention. It was unfortunate that the training was implemented at the same time as major changes in the national aged care standards were introduced. Despite nursing homes being aware of the new standards, they were not prepared for the amount of additional training and administrative work it entailed. Several other barriers to IMPETUS-D uptake were identified and related to staff (turnover and high workload), organisational factors (communication, scheduling disruptions), and the characteristics of the program (technical issues, its voluntary nature, some modules too long). Strategies such as having the IMPETUS-D training integrated into the organisation's training schedule and available on the organisation's learning management system may have helped overcome some of these barriers and increased uptake. However, many of these challenges have been reported in other nursing home-based studies [27, 28], and highlight the need for future research to evaluate the effect of strategies to address common barriers.

A pilot intervention phase was not undertaken due to time and resource constraints. This may have aided identification of implementation barriers. However, a pilot phase may not have mitigated unexpected system changes which were beyond researcher control. Further, the investigators considered that the bespoke design of the training package and high-level stakeholder buy-in and engagement with the training package with all homes being managed by the same private aged care provider would support staff access to and uptake of training. Unfortunately, in this study, this was not sufficient in and of itself to ensure staff participation and further research is needed to better understand nursing home context specific barriers and strategies to overcome these.

This study set out to evaluate the effectiveness of the IMPETUS-D intervention, but without sufficient staff participation (implementation failure), we were unable to establish the true effect of the training on staff knowledge, practice, and resident outcomes. Future work in this area might benefit from a more detailed assessment of individual care homes' readiness to implement the training with input from general managers, nurses, PCWs, residents, and their families. This information could then be used to target care homes that are ready to implement the training, and to inform the research or implementation team on how best to support implementation, based on the local needs. 


\section{Conclusion}

Our study of a staff training program on palliative dementia care was unable to show any differences in hospital transfers or staff knowledge and attitudes. Staff participation was lower than expected and highlighted the need to ensure nursing homes are ready to implement the intervention. Training programs for nursing home staff are important and necessary as the population ages and the prevalence of dementia increases but more research is required to identify effective strategies to address barriers to implementation in these settings

\section{Abbreviations}

ACD Advance care directive

ACFI Aged Care Funding Instrument

ANZCTR Australia and New Zealand Clinical Trial Register

CFIR Consolidated Framework for Implementation Research

GOC Goals of Care

HREC Human Research and Ethics Committee

ICC Intra-Cluster Correlation

IMPETUS-D IMproving Palliative care Education and Training Using Simulation in Dementia

ITT Intention to treat

LMS Learning management system

OR Odds ratio

PCWs Personal care workers

RCT Randomised controlled trial

\section{Declarations}

\section{Ethics and consent}

Ethics approval was received by the Melbourne Health Human Research and Ethics Committee (HREC/17/MH/336). All methods were performed in accordance with the National Statement of Ethical Conduct in Research Involving Humans, and the research arrangements of the organisations involved. 


\section{Consent to participate}

Consent for participation in IMPETUS-D and the associated evaluation was conducted at the organisation level. Consent from individuals living in the residential aged care facilities has been waived, as it was deemed unnecessary by the Melbourne Health Human Research and Ethics Committee. Individual consent from residential aged care staff who participated in the research was sought. Invitations to participate in the bereaved family carer survey and information about the project was provided to family members, and completion and return of the survey implied consent.

\section{Patient safety}

The number of deaths and hospital transfers was monitored in the control and intervention groups. The plain language statements included contact details of the principal investigator, project coordinator and the ethics committee that participants can contact in the event of a complaint or concern about the project. Any reported issues, adverse events and other unintended effects of the trial intervention were reported to the IMPETUS-D Project Working and Advisory Group and the IMPETUS-D Steering Committee.

\section{Study management and oversight}

This study was managed by the IMPETUS-D Project Working and Advisory Group, consisting of the principal investigator, co-investigators and representation from the aged care home provider. The IMPETUS-D Steering Committee with members from stakeholder groups provided additional governance oversight for the study monitoring.

Written 6-monthly progress reports were submitted to the funding body and annual progress reports submitted to the Melbourne Health ethics committee throughout the project timeline.

\section{Consent for publication}

Not applicable.

\section{Availability of data and materials}

Data will be available after analyses is finalised and report / publication has been submitted and approved. Unidentifiable individual participant data and related data dictionaries will be available. Access is subject to approval by the Principal Investigator Professor Wen Kwang Lim.

\section{Competing interests}

The authors declare that they have no competing interests.

\section{Funding}

This work was supported by funding from the Australian Government Department of Health under the Dementia and Aged Care Services Grant Opportunity 1 . The funders did not have any input in the study 
design, analysis, or manuscript development. Joanne Tropea received a Melbourne Ageing Research Collaboration (MARC) PhD scholarship.

\section{Author contributions}

JT wrote the main manuscript, JT and GH conducted the statistical analysis, and JT, CAB and BJH contributed to the analysis of the qualitative data. CEJ, DN, BJH, WKL, RB and JT developed the training intervention. All authors contributed design of the evaluation and the interpretation of findings and reviewed the manuscript.

Acknowledgements: The authors acknowledge the IMPETUS-D Working Group and Steering Committee, including Lee-Anne Henley, Liz Reymond, Stephen Wiblin, Margaret Winbolt, David Currow, Robyn Smith, Anita Goh, and Lyn Franco. Special thanks to Marg Ryan, Arlene Nunez, Gurleen Kaur, Amanda Smith, Kristine Min and all the staff and family participants.

\section{References}

1. Australian Institute of Health and Welfare. Interfaces between aged care and health systems in Australia-where do older Australians die? 2021 [29/07/2021]. Available from: https://www.aihw.gov.au/reports/aged-care/where-do-older-australians-die/summary.

2. Martinsson L, Lundstrom S, Sundelof J. Quality of end-of-life care in patients with dementia compared to patients with cancer: A population-based register study. PLoS One. 2018; 13(7): e0201051. doi: 10.1371/journal.pone.0201051.

3. Mitchell SL, Kiely DK, Hamel MB. Dying with advanced dementia in the nursing home. Arch Intern Med. 2004; 164(3): 321-6. doi: 10.1001/archinte.164.3.321.

4. Palliative Care Australia and Alzheimer's Australia. End of life care for people with dementia. Survey Report 2014. 2014.

5. Commonwealth of Australia. Royal Commission into Aged Care Quality and Safety. Final Report: Care, Dignity and Respect. Volume 1. Summary and Recommendations. 2021.

6. Worldwide Hospice Palliative Care Alliance (WHPCA). Palliative Care and Dementia Statement Worldwide Hospice Palliative Care Alliance. 2015 [9 August 2019]. Available from: https://www.who.int/mental_health/neurology/dementia/NSA_WHPCA.pdf.

7. Chang E, Daly J, Johnson A, et al. Challenges for professional care of advanced dementia. Int J Nurs Pract. 2009; 15(1): 41-7. doi: 10.1111/j.1440-172X.2008.01723.x.

8. Tropea J, Bicknell R, Nestel D, et al. Simulation training in non-cancer palliative care for healthcare workers: a systematic review of controlled studies. BMJ Simulation and Technology Enhanced Learning. 2021; 7(4): 262-269. doi: 10.1136/bmjstel-2019-000570.

9. Arcand $\mathrm{M}$, Monette $\mathrm{J}$, Monette $\mathrm{M}$, et al. Educating nursing home staff about the progression of dementia and the comfort care option: impact on family satisfaction with end-of-life care. J Am Med Dir Assoc. 2009; 10(1): 50-5. doi: 10.1016/j.jamda.2008.07.008. 
10. Caplan GA, Meller A, Squires B, Chan S, Willet W. Advance care planning and hospital in the nursing home. Age and Ageing. 2006; 35: 581-5.

11. Kortes-Miller K, Jones-Bonofiglio K, Hendrickson S, Kelley ML. Dying With Carolyn: Using Simulation to Improve Communication Skills of Unregulated Care Providers Working in Long-Term Care. J Appl Gerontol. 2016; 35(12): 1259-1278. doi: 10.1177/0733464815577139.

12. Livingston G, Lewis-Holmes $E$, Pitfield $C$, et al. Improving the end-of-life for people with dementia living in a care home: an intervention study. Int Psychogeriatr. 2013; 25(11): 1849-58. doi: $10.1017 /$ S1041610213001221.

13. Verreault R, Arcand M, Misson L, et al. Quasi-experimental evaluation of a multifaceted intervention to improve quality of end-of-life care and quality of dying for patients with advanced dementia in long-term care institutions. Palliat Med. 2018; 32(3): 613-621. doi: 10.1177/0269216317719588.

14. Tropea J, Johnson CE, Nestel D, et al. A screen-based simulation training program to improve palliative care of people with advanced dementia living in residential aged care facilities and reduce hospital transfers: study protocol for the IMproving Palliative care Education and Training Using Simulation in Dementia (IMPETUS-D) cluster randomised controlled trial. BMC Palliat Care. 2019; 18(1): 86. doi: 10.1186/s12904-019-0474-x.

15. Campbell MK, Piaggio G, Elbourne DR, Altman DG, Group C. Consort 2010 statement: extension to cluster randomised trials. BMJ. 2012; 345: e5661. doi: 10.1136/bmj.e5661.

16. Harris PA, Taylor R, Thielke R, Payne J, Gonzalez N, Conde JG. Research electronic data capture (REDCap): a metadata-driven methodology and workflow process for providing translational research informatics support. J Biomed Inform. 2009; 42(2): 377-81. doi: 10.1016/j.jbi.2008.08.010.

17. Long CO, Sowell EJ, Hess RK, Alonzo TR. Development of the questionnaire on palliative care for advanced dementia (qPAD). Am J Alzheimers Dis Other Demen. 2012; 27(7): 537-43. doi: $10.1177 / 1533317512459793$.

18. Kiely DK, Volicer L, Teno J, Jones RN, Prigerson HG, Mitchell SL. The validity and reliability of scales for the evaluation of end-of-life care in advanced dementia. Alzheimer Dis Assoc Disord. 2006; 20(3): 176-81. doi: 10.1097/00002093-200607000-00009.

19. StataCorp. Stata Statistical Software: Release 15., College Station, TX: StataCorp LLC. 2017.

20. NVivo qualitative data analysis Software; QSR International Pty Ltd. Version 122018.

21. Liamputtong P. Research methods in health : Foundations for evidence based practice. 2013, ProQuest Ebook Central.

22. Martin RS, Hayes BJ, Hutchinson A, Tacey M, Yates P, Lim WK. Introducing Goals of Patient Care in Residential Aged Care Facilities to Decrease Hospitalization: A Cluster Randomized Controlled Trial. J Am Med Dir Assoc. 2019; 20(10): 1318-1324.e2. doi: 10.1016/j.jamda.2019.06.017.

23. CFIR Research Team-Center for Clinical Management Research. Consolidated Framework for Implementation Research. 2021 [08/10/2021]. Available from: https://cfirguide.org/.

24. Lamppu PJ, Finne-Soveri H, Kautiainen H, Laakkonen ML, Laurila JV, Pitkala KH. Effects of Staff Training on Nursing Home Residents' End-Of-Life Care: A Randomized Controlled Trial. J Am Med Dir 
Assoc. 2021doi: 10.1016/j.jamda.2021.05.019.

25. Van den Block L, Honinx E, Pivodic L, et al. Evaluation of a Palliative Care Program for Nursing Homes in 7 Countries: The PACE Cluster-Randomized Clinical Trial. JAMA Intern Med. 2020; 180(2): 233-242. doi: 10.1001/jamainternmed.2019.5349.

26. Oosterveld-Vlug M, Onwuteaka-Philipsen B, Ten Koppel M, et al. Evaluating the implementation of the PACE Steps to Success Programme in long-term care facilities in seven countries according to the RE-AIM framework. Implement Sci. 2019; 14(1): 107. doi: 10.1186/s13012-019-0953-8.

27. Lam HR, Chow S, Taylor K, et al. Challenges of conducting research in long-term care facilities: a systematic review. BMC Geriatr. 2018; 18(1): 242. doi: 10.1186/s12877-018-0934-9.

28. Low LF, Fletcher J, Goodenough B, et al. A Systematic Review of Interventions to Change Staff Care Practices in Order to Improve Resident Outcomes in Nursing Homes. PLoS One. 2015; 10(11): e0140711. doi: 10.1371/journal.pone.0140711.

\section{Figures}




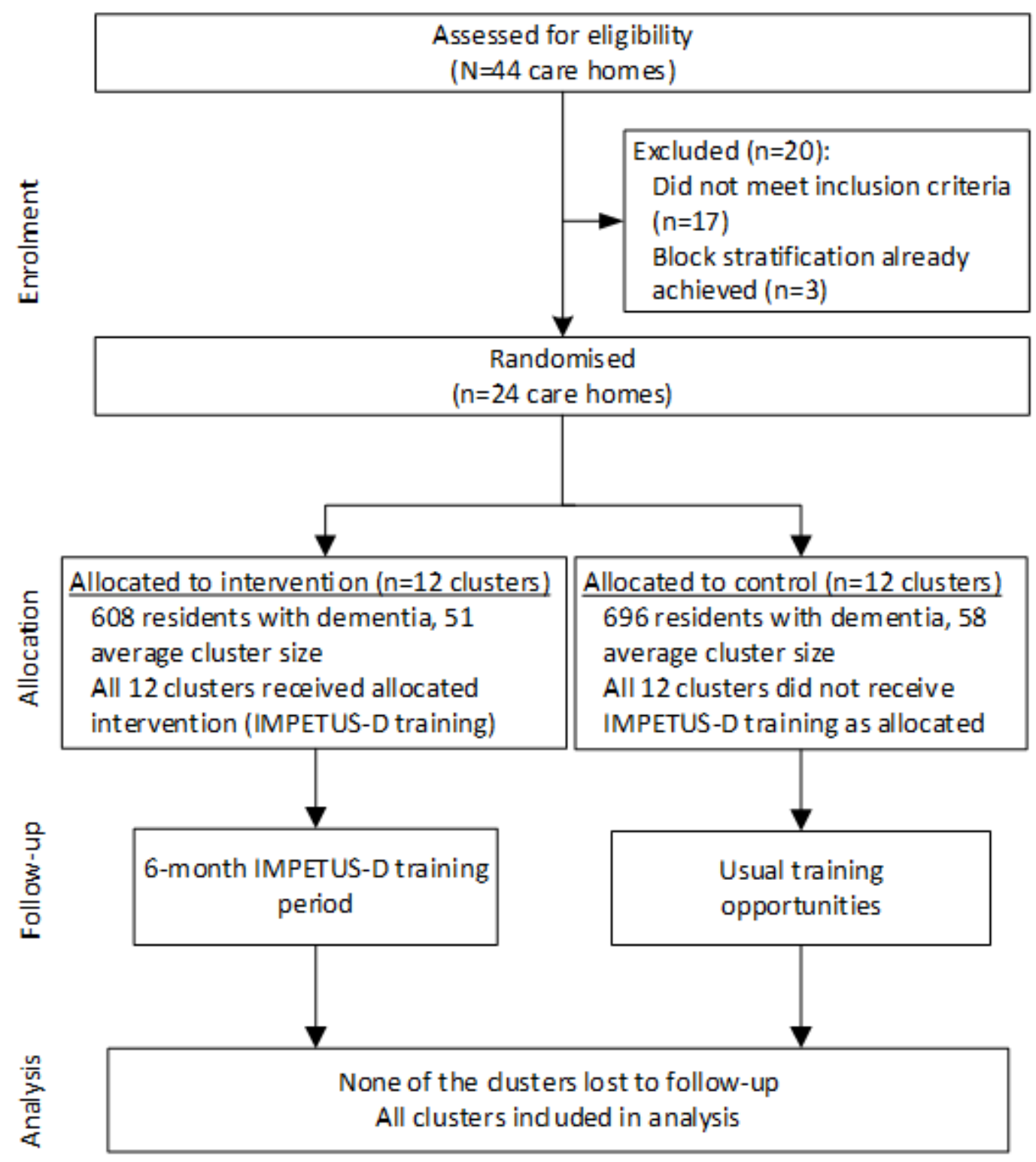

\section{Figure 1}

Study flowchart 


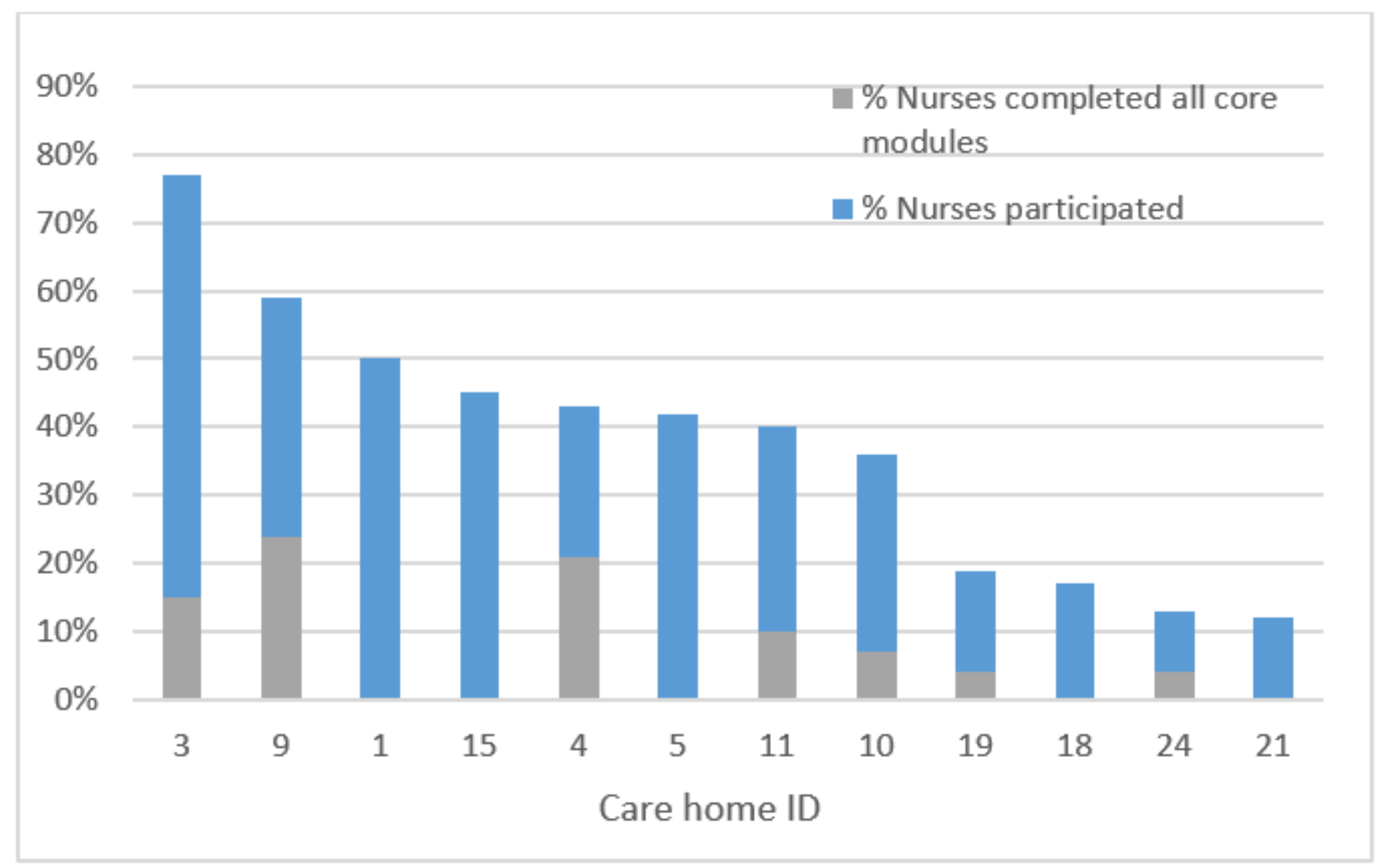

Figure 2

Percentage of nurses who completed core modules or participated by care home

\section{Supplementary Files}

This is a list of supplementary files associated with this preprint. Click to download.

- Supplementarymaterial1.docx 